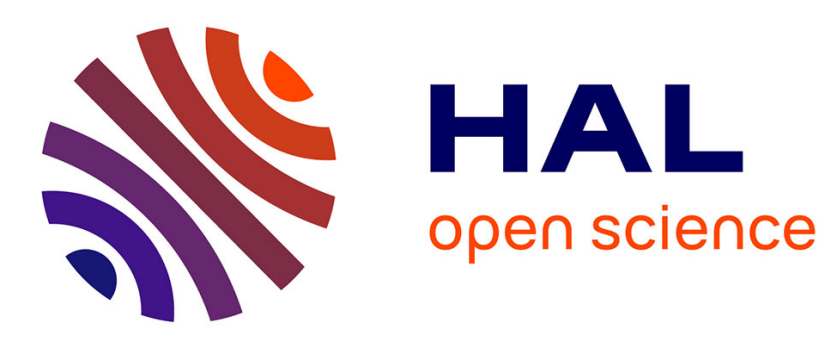

\title{
Status of orthopositronium decay rate measurements D. Sillou
}

\section{To cite this version:}

D. Sillou. Status of orthopositronium decay rate measurements. International Workshop on Positronium Physics, May 2003, Zurich, Switzerland. pp.3919-3925, 10.1142/S0217751X04020178 . in2p300014121

\section{HAL Id: in2p3-00014121 \\ https://hal.in2p3.fr/in2p3-00014121}

Submitted on 14 Nov 2003

HAL is a multi-disciplinary open access archive for the deposit and dissemination of scientific research documents, whether they are published or not. The documents may come from teaching and research institutions in France or abroad, or from public or private research centers.
L'archive ouverte pluridisciplinaire $\mathbf{H A L}$, est destinée au dépôt et à la diffusion de documents scientifiques de niveau recherche, publiés ou non, émanant des établissements d'enseignement et de recherche français ou étrangers, des laboratoires publics ou privés. 
LAPP-EXP 2003-08

November 2003

\title{
Status of Orthopositronium Decay Rate Measurements
}

\author{
D. Sillou \\ LAPP-IN2P3-CNRS \\ 9 chemin de Bellevue - BP. 110, F-74941 Annecy-le-Vieux Cedex
}

Presented at the International Workshop on Positronium Physics

ETH Zurich, May 30-31, 2003 


\title{
Status of Orthopositronium Decay Rate Measurements
}

\author{
SILLOU D.
}

LAPP - 9 Chemin de Bellevue - BP 11074941 Annecy-le-Vieux CEDEX - FRANCE email: daniel.sillou@lapp.in2p3.fr

The so-called orthopositronium lifetime puzzle have existed from more than 10 years. Recent measurements are now in good agreeement with the predicted values. Should we consider that the problem is solved now as claimed in a recent paper? We present a short review of the experiments during the past 15 years.

Talk presented at the International Workshop on Positronium Physics ETH Zurich 30-31 May 2003

\section{Introduction}

Positronium was discovered in 1951 by Deutsch ${ }^{1}$ who observed its decays in different gases. A history of the experimental and theoretical works, especially concerning the orthopositronium (oPs) lifetime measurements, may be found in reference ${ }^{2}$ and in references therin. The evolutions of both theory and experiment are summarized in fig. 1.

As shown in this picture, the precision of the measurements has isteadily mproved since $1989^{3}$. For this reason we will limit ourself to the results posterior to this

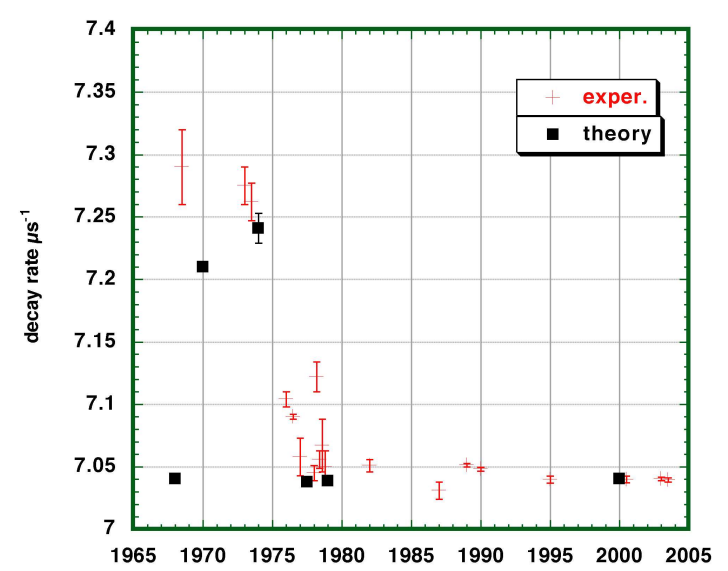

Fig. 1. history of oPs decay rate results. 


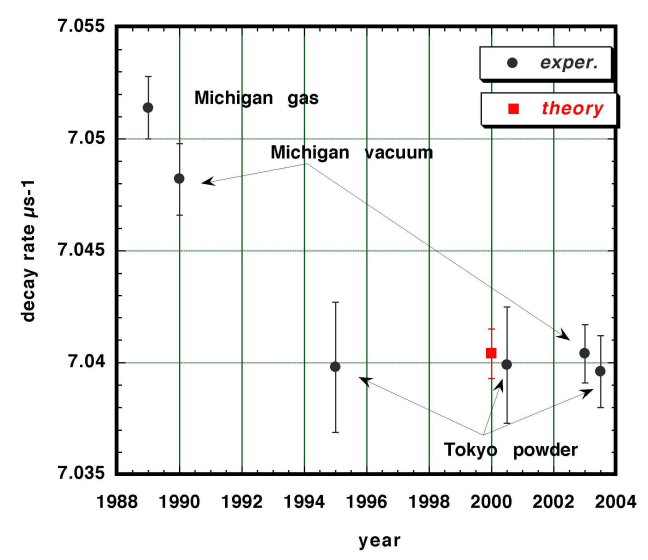

Fig. 2. history of recent oPs decay rate results.

date which display errors well under 1000 ppm (see fig. 2).

The oPs lifetime puzzle originates from the 1989 and $19900^{3}, 4$ measurements of the Michigan group which disagree with theoretical predictions. Nevertheless, one could have expected at this time that an unforeseen huge contribution from higher order terms would help in solving the problem. The computation of the oPs lifetime to order $\alpha^{2}{ }^{2}$, put a final point on this expectation and brought a new interest to the oPs lifetime problem.

Because of these two discrepant results much of experimental activity has been devoted to the clarification of the oPs lifetime puzzle (see reference ${ }^{6}$ and references therein) which includes:

- Direct lifetime measurements in 1995 which do not confirm the discrepancy.

- Searches of new visible oPs decay channels into $1,2 \gamma$, high decay rate into $5 \gamma, 1$ gamma and one new boson ...

- Suggestions for Ps disappearance mechanisms (Mirror world, millicharged particles, extra-dimensions...)

Several experiments quoted below, have also contributed to clarify experimental difficulties. Recent results using different experimental techniques are now in agreement with the predicted values. Should we consider that the oPs lifetime puzzle is solved now or does it require additional experimental investigations?

\section{Review of the experimental results.}

As stressed above, we will limit ourselves to experiments posterior to 1989. From this time, six results were published by two groups : Michigan University and 
Tokyo University. The results are summarized in table 1 which shows the initial discrepancy and the present agreement with theory. The first Michigan experiment was performed in gas and the later experiments in vacuum. The

Tokyo experiments were all performed in powders.

Table 1. Experimental results and theory

\begin{tabular}{ccccccc}
\hline Year & group/ref & $\begin{array}{c}\text { Rate } \\
\mu s^{-1}\end{array}$ & $\begin{array}{c}\text { errors } \\
(\mathrm{ppm})\end{array}$ & Technique & exp - th & $\begin{array}{c}\text { exp - th } \\
\text { (sigma) }\end{array}$ \\
\hline 1989 & Michigan [3] & 7.0514 & 200 & Gas & .01142 & 8.2 \\
1990 & Michigan[4] & 7.0482 & 230 & Vacuum & .00822 & 5.1 \\
1995 & Tokyo [ $\left.{ }^{5}\right]$ & 7.0398 & 412 & Powder & -.00018 & -.06 \\
2000 & Tokyo [ $\left.{ }^{7}\right]$ & 7.0399 & 412 & Powder & -.00008 & 0.0 \\
2003 & Michigan $\left[{ }^{8}\right]$ & 7.0404 & 185 & Vacuum & .00042 & 0.32 \\
2003 & Tokyo $\left[{ }^{9}\right]$ & 7.0396 & 227 & Powder & -.00038 & .024 \\
2000 & {$\left[^{2}\right]$} & 7.039979 & 1.6 & Theory & & \\
\hline
\end{tabular}

\section{The experiments}

The experimental problems differ somewhat with the technique used.

\subsection{The 1989 gas experiment.}

In this experiment ${ }^{3}$, the oPs are produced by stopping positrons from a ${ }^{22} \mathrm{Na}$ source in a gas chamber where they are confined. Collisions of oPs with in the gas molecules result in pickoff interactions and an extrapolation to zero pressure was done in order to eliminate this pickoff. These extrapolations were performed in four different gases measurements and the convergence to a single value was very good. Nevertheless, the extrapolations assume that the oPs has thermalised by collisions with gas molecules independently of the gas pressure. This assumption was shown to be incorrect in 1998 by the same group ${ }^{10}$ using the Doppler Broadening Spectroscopy technique. Thus t, he validity of the extrapolation was questionable and this could explain the disagreement with the prediction.

\subsection{Vacuum experiments.}

These experiments ${ }^{4,8}$ make use of a more sophisticated setup designed to take into account experimental problems of previous 1989 measurements]. The positrons, from a slow positron beam, impact on a layer in which positronium are formed and are confined in a cavity surrounding the formation region. In this setup the pickoff rate (which was much lower than in the gas) and the possibility for the positronium to escape through the entrance aperture of the cavity had to 


\subsubsection{The 1990 vacuum experiment.}

The oPs were produced in a fumed $\mathrm{MgO}$ layer with $700 \mathrm{eV}$ incident positrons. Measurements were performed with cavities of different sizes and a two variables extrapolation as a function of the cavity size and of the ratio of the entrance aperture to the cavity size was performed. One remaining question concerns the possibility that positronium could remain trapped in pores of $\mathrm{MgO}$ which could explain the presence of an unexplained component with a 40ns lifetime in the decay rate spectrum. This point has been carefully studied and ruled out, as being the origin of the discrepancy, at the level of $80 \mathrm{ppm}$ (much less than the 1000 ppm level of discrepancy itself).

\subsubsection{The 2003 vacuum experiment.}

The experiment was performed with a similar setup improved on many points concerning in particular:

- The positronium was formed in a porous silica film rather than in $\mathrm{MgO}$. In the pore of this film, the positronium energy spectrum approach thermalisation ${ }^{11}$.

- The beam energy tuning to control precisely the implantation energy of positrons in the porous film.

- A double confinement cavity which reduces strongly the oPs escape through the entrance aperture of the cavity.

This first improvement was done in order to suppress the problems due to positronium trapping in pores inside the $\mathrm{MgO}$ layer, quoted above. The positronium energy spectrum shows that the positronium are essentially

thermalized, but it includes a high energy tail with two additional components: an epithermal components extending till $2 \mathrm{eV}$ and a component due to backscattered positrons which peaks up an electron near the surface of the film. This component extends till $20 \mathrm{eV}$.

The contribution of these non-thermalized components had to be taken into acccount. This was done in independent measurements performed with a non porous film, so that the low energy component is suppressed. The amount of this suppression is controlled by tuning the positron beam implantation energy ${ }^{12}$. To exclude the effects of these two high energy components, measurements were performed with porous and non-porous films at several beam energy implantation

in the range 1 to $5 \mathrm{keV}$. Figure 3 shows the reduction of the high energy component in function of the beam energy. It shows also the computed decay rate as a function of the starting time of the fit .

An extrapolation procedure was performed in order to extract the decay rate at zero backscattered oPs, in two ways:

- By extrapolation from a fit of the full spectrum. 

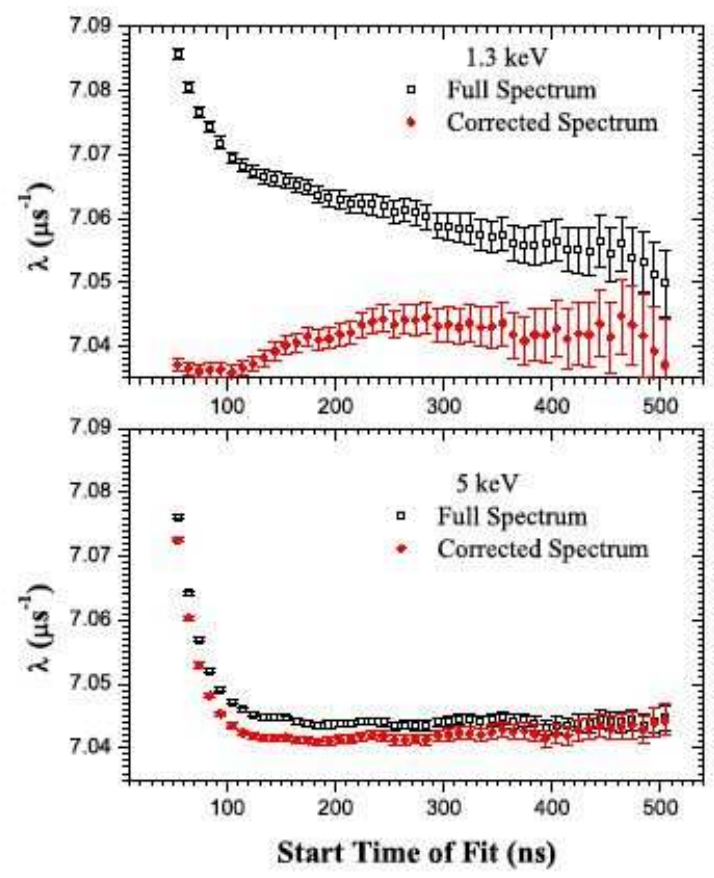

Fig. 3. Decay rate in function of the starting time of the fit for different implantation energy, for the corrected and non corrected spectrum.

- By extrapolation of the spectrum corrected by the non porous film measurements.

These two extrapolation converge to a single value when the backscattered component is zero (fig. 4 ).

An analysis of the former vacuum experiment leads the authors to conclude that in the 1990 measurementss they did not take properly into account the "backscattered" high energy positronium component. The contribution of this component did exist for large cavities size and is probably the origin of the discrepancy.

\subsection{The powder experiments.}

The Tokyo experiments ${ }^{5}, 7,9$ have been performed by using positrons from a

(22)Na source which form positronium in $\mathrm{SiO}_{(2)}$ powders. The setups are essentially the same apart from improvements in scintillators, electronic.... The pickoff rate is much higher than in the vacuum experiments but it is measured directly using a high resolution Germanium detector which identify the $511 \mathrm{keV} \gamma$ on the high energy side of the three $\gamma$ spectrum (see figure 5 ). 


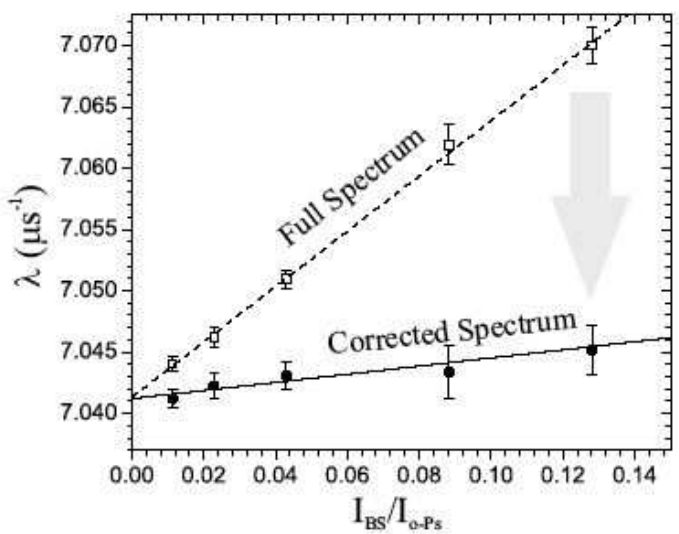

Fig. 4. Extrapolation to "zero backscattered" positronium for both corrected and non corrected spectrum.

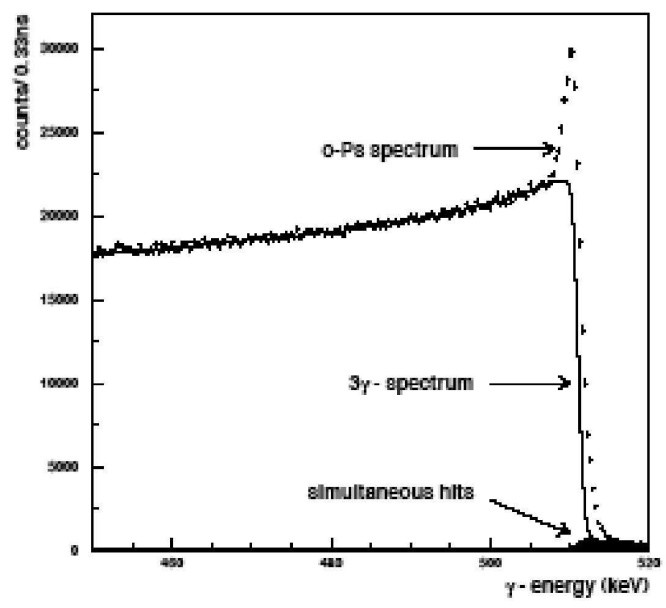

Fig. 5. Three $\gamma$ from oPs decay and $.511 \mathrm{keV}$ line from $2 \gamma$ decay in the Ge detector.

This allows for a precise evaluation of the pickoff contribution as shown in fig. 6 for two different size of the grain of the powders.

\section{Discusssion.}

The Michigan group has performed an extensive work to clarify the problem of thermalization of the oPs in gas to understand the origin of the discrepancy in the 


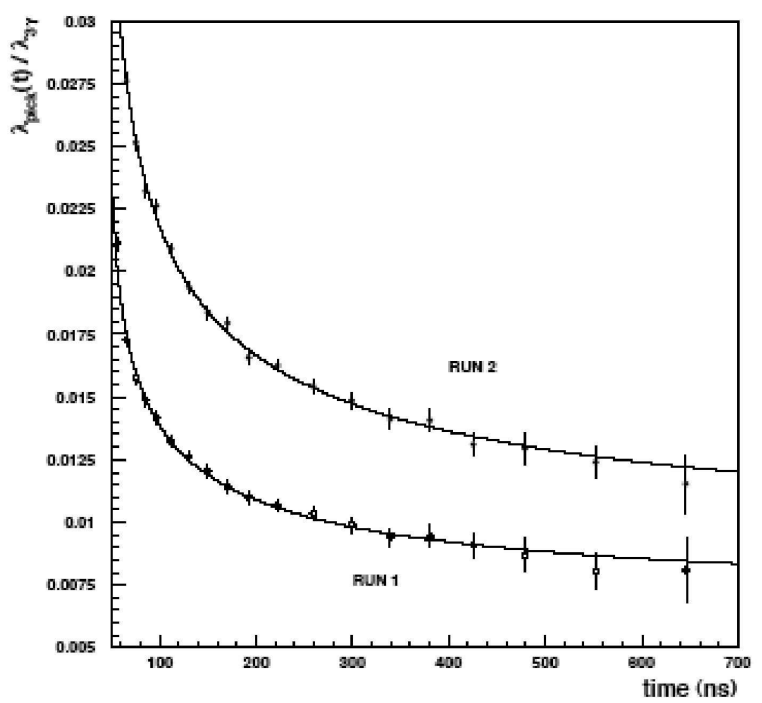

Fig. 6. Relative pickoff rate in function of time.

1989 experiment. The 1990 vacuum experiment could suffer from the presence of backscattered high energy positronium. If this effect would represent the major source of the discrepancy, it should contribute at the level of $1100 \mathrm{ppm}$.

Surprisingly, one does not observe, at this level, $2 \gamma$ decay due to pickoff ${ }^{15}$ which is excluded at the level of 100ppm in an experiment searching for a $2 \gamma$ decay channel of the oPs with the same setup ${ }^{16}$. The powder experiments present the advantage to measure directly the pickoff correction. They have been subject to questions concerning the possibility that charging effects in the powders could perturbate the oPs lifetime. The answer to this question by the Tokyo group both by an evaluation of the effect and by measurements using different powders, which are in extremely good agreement with each others, seems convincing. Nevertheless one should also remember that some mechanisms invoked to explain the oPs decay rate discrepancy such as an oscillation mechanism between positronium and mirror-positronium would be suppressed in matter due to the high collision rate which would destroy any coherence effect?

\section{Conclusions.}

Recent oPs decay rate results show an agreement between experiment and theory.

Nevertheless these experiments are difficult as showed past discrepancies and some experimental facts are not fully understood (as for instance the absence of 2

$\gamma$ in the first Michigan vacuum experiment). The powder experiments are in extremely good agreement with theory nevertheless the presence of matter could 


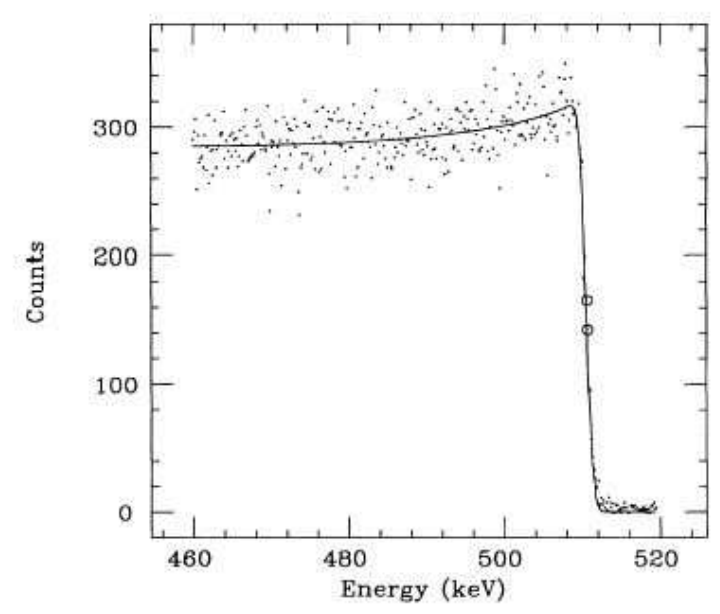

Fig. 7. Three $\gamma$ from oPs decay in search of $2 \gamma$ decay in the Ge detector.

prevent some invisible decay mechanism. Finally the theoretical prediction could require addditional corrections ${ }^{13}$ but the final result will reach a precision of the order of 1-2 ppm while experimental errors are two order of magnitude higher

( 200ppm). For these reasons, new experiments to search for invisible decays or to measure the oPs decay rate in vacuum using a technique allowing for direct pickoff measurement, with lower systematics would bring valuable informations.

The feasibility of such experiments are under studies ?.

\section{References}

1. M. Deutsch, Phys. Rev. 82, 455 (1951).

2. G. Adkins, R.N. Fell, J. Sapirstein, Annals of Physics 295, 136-193 (2002).

3. C.I. Westbrook, D.W. Gidley, R.S. Conti and A. Rich, Phys. Rev. A40, 5489 (1989).

4. J.S. Nico, D.W. Gidley and A. Rich, Phys. Rev. Lett. 65, 1344 (1990).

5. S. Asai, S. Orito and N. Shinohara, Phys. Lett. B 357, 475-480 (1995).

6. M.I. Dobrolioubov, S.N. Gninenko, A. Yu Ignatiev, V. A. Matveev, J. Mod. Phys. A8, 2859 (1993).

M.Skalsey , Mater. Science Forum Trans Tech Pub. Switzerland. 209, 255-257 (1997). S.N. Gninenko, N.V. Krasnikov and A. Rubbia, Mod. Phys. Lett. A17 1713 (2002).

7. O. Jinnouchi, S. Asai and T. Kobayashi, hep-ex/0011011.

8. R.S. Vallery, P.W. Zitzewitz and D.W. Gidley, Phys. Lett. 90, (2003).

9. O. Jinnouchi, S. Asai and T. Kobayashi, Preprint submitted to Elsevier Science. PACS: 36.10.Dr, 11.10. St., (2003).

10. M. Skalsey, J. J. Engbrecht, R. K. Bithell, R. S. Vallery, and D. W. Gidley, Phys. Rev. Lett. 80, 17 (1998).

11. D.W. Gidley, W.E. Frieze, T.L. Dull, A. F. Yee, E.T. Ryan and H.-M. Ho , Phys. Rev. B 60, 5157 (1999).

12. D.W. Gidley, D.N. McKinsey and P.W. Zitzewitz, J. Appl. Phys. 78, 1406 (1995).

13. G. Lopez Castro, L. Pesstieau and C. Smith, hep-ph 0004209, (2000) 
November 5, $2003 \quad$ 9:14 LAPP-EXP 2003-08

OPs decay rate experiments. 9

14. Paul A. Vetter, Int. J. Mod. Phys. A

15. S. Gninenko, discussion at the Workshop on Positronium Physics, ETH Zurich 30-31 may 2003.

16. D.W.Gidley, J.S. Nico and M. Skalsey, Phys. Rev. Lett. 66, 1302-1305 (1991).

17. R. Foot, S.N. Gninenko, Phys lett. B 480, 171-175 (2000).

18. P. Vetter contribution to this workshop. 